Supplemental Material

\title{
Comparing the Pfizer Central Nervous System Multiparameter Optimization Calculator and a BBB Machine Learning Model
}

\author{
Fabio Urbina ${ }^{\$, \dagger}$, Kimberley M. Zorn ${ }^{\dagger}$, Daniella Bruner ${ }^{\$}$ and Sean Ekins ${ }^{{ }^{\star}}$
}

\$Department of Cell Biology and Physiology, University of North Carolina at Chapel Hill, Chapel Hill, NC 27599-7545, USA

†Collaborations Pharmaceuticals, Inc., 840 Main Campus Drive, Lab 3510, Raleigh, NC 27606, USA.

\$PsychoGenics, 215 College Road, Paramus, NJ 07652, USA

*To whom correspondence should be addressed. E-mail: sean@collaborationspharma.com

Phone: $215-687-1320$ 


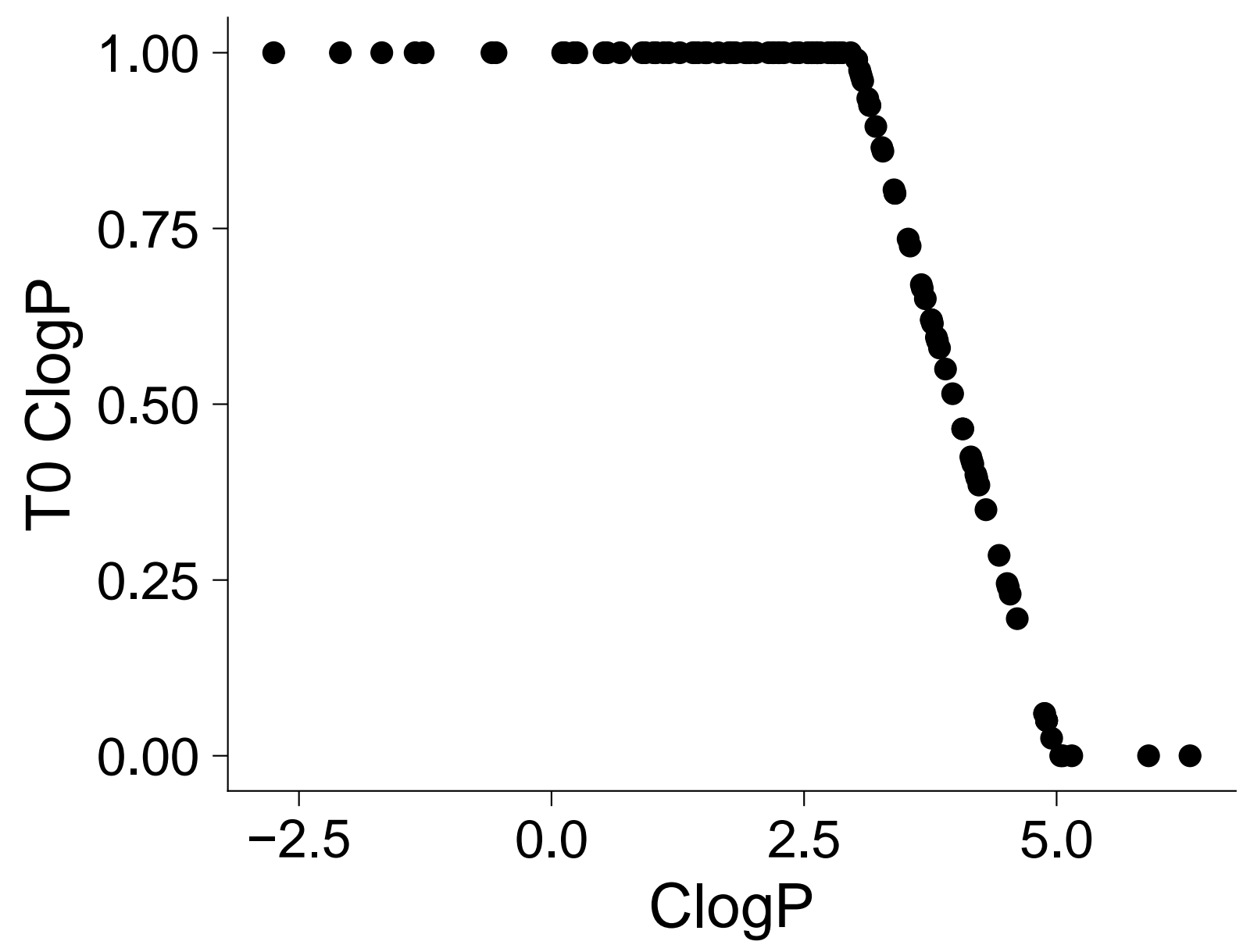

Figure S1. ClogP and T0 ClogP plot from CPI which can be compared with that previously published by Wager et al., $2010^{1}$. 

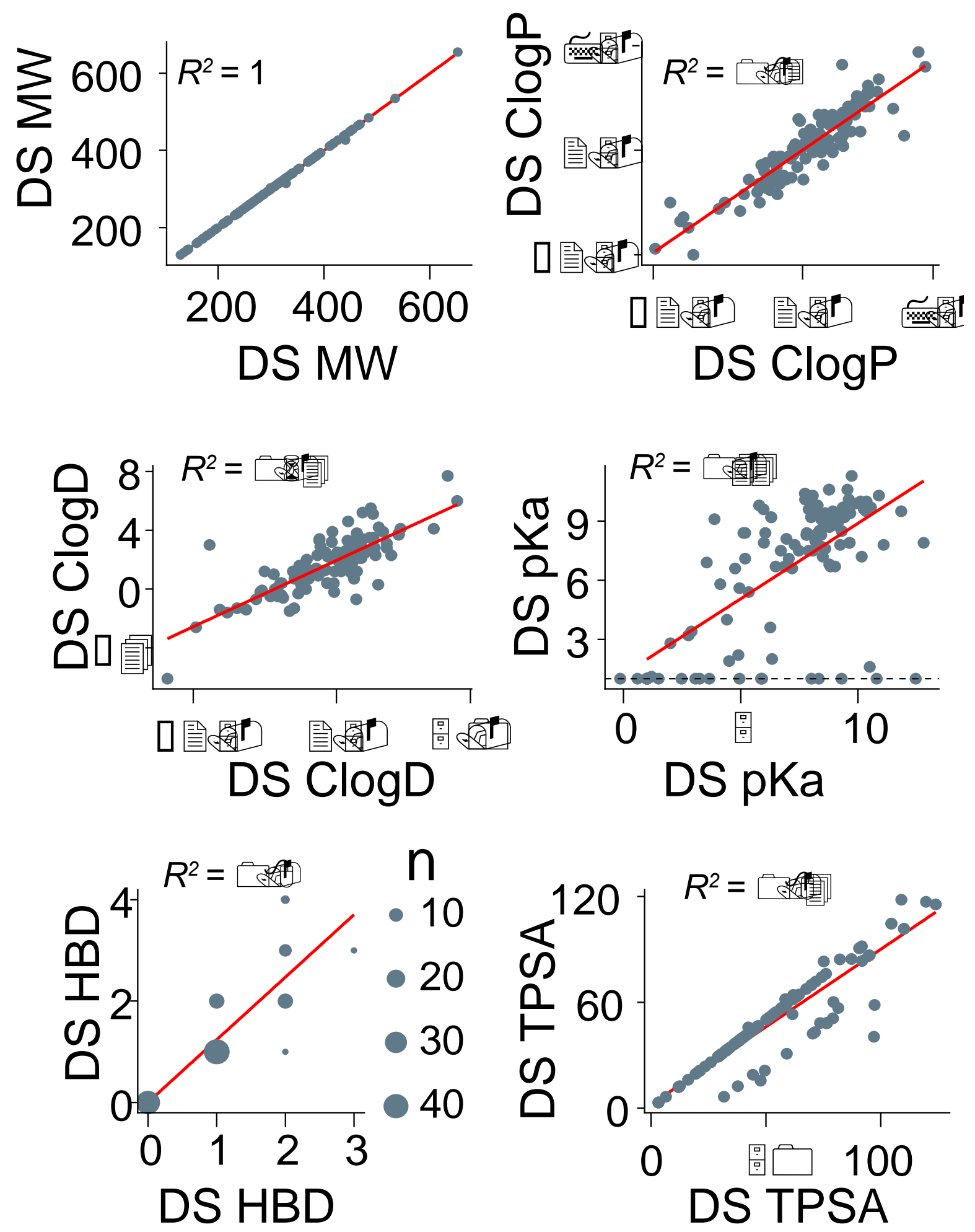

Figure S2. Set of descriptors generated from Discovery Studio (DS) vs. Pfizer, using the same chemical structures $(P<<0.005)$. 


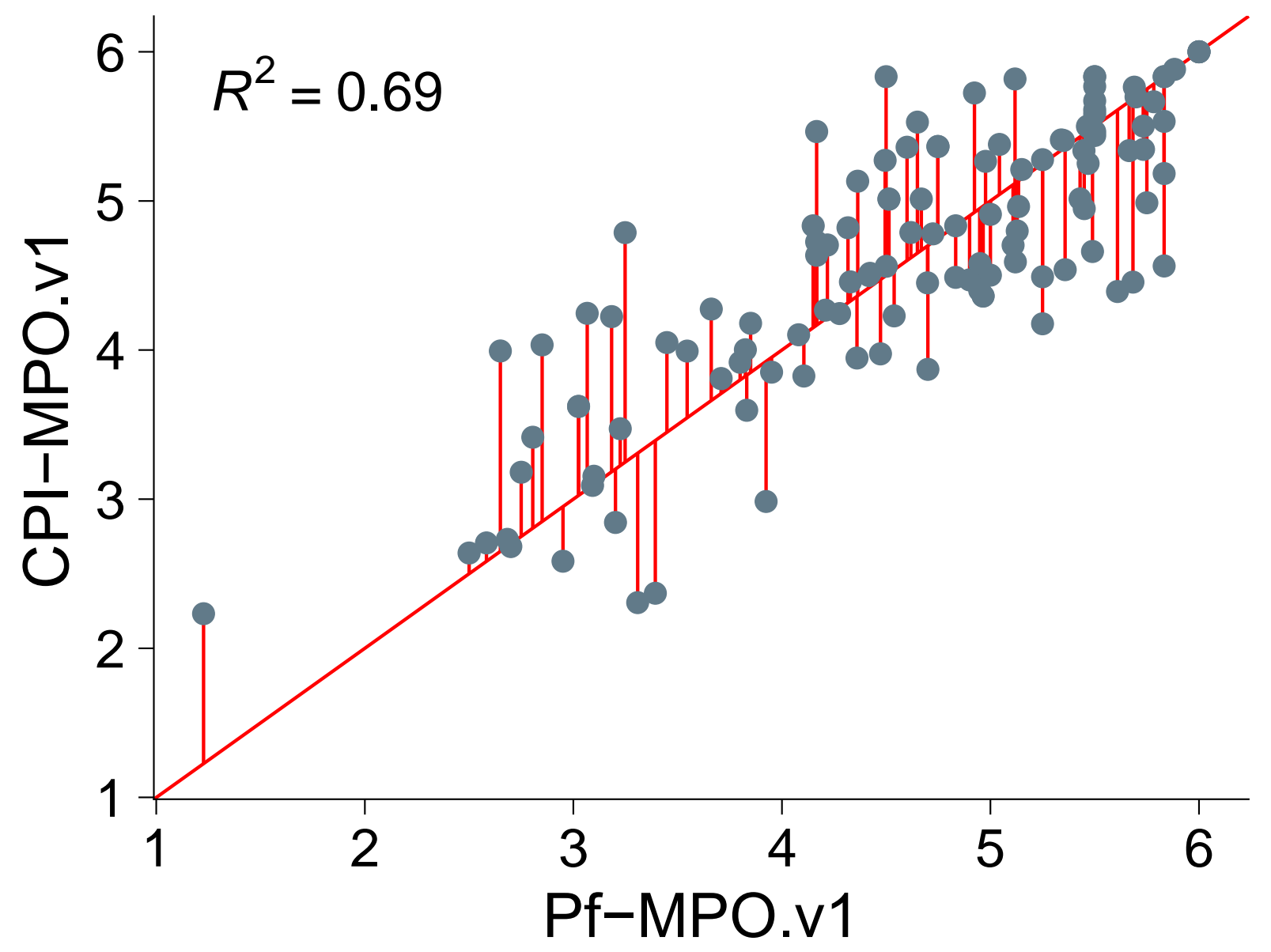

Figure S3. Correlation between MPO score calculated with Discovery Studio (DS) descriptors and Pfizer MPO score ( $P<0.005)$. 


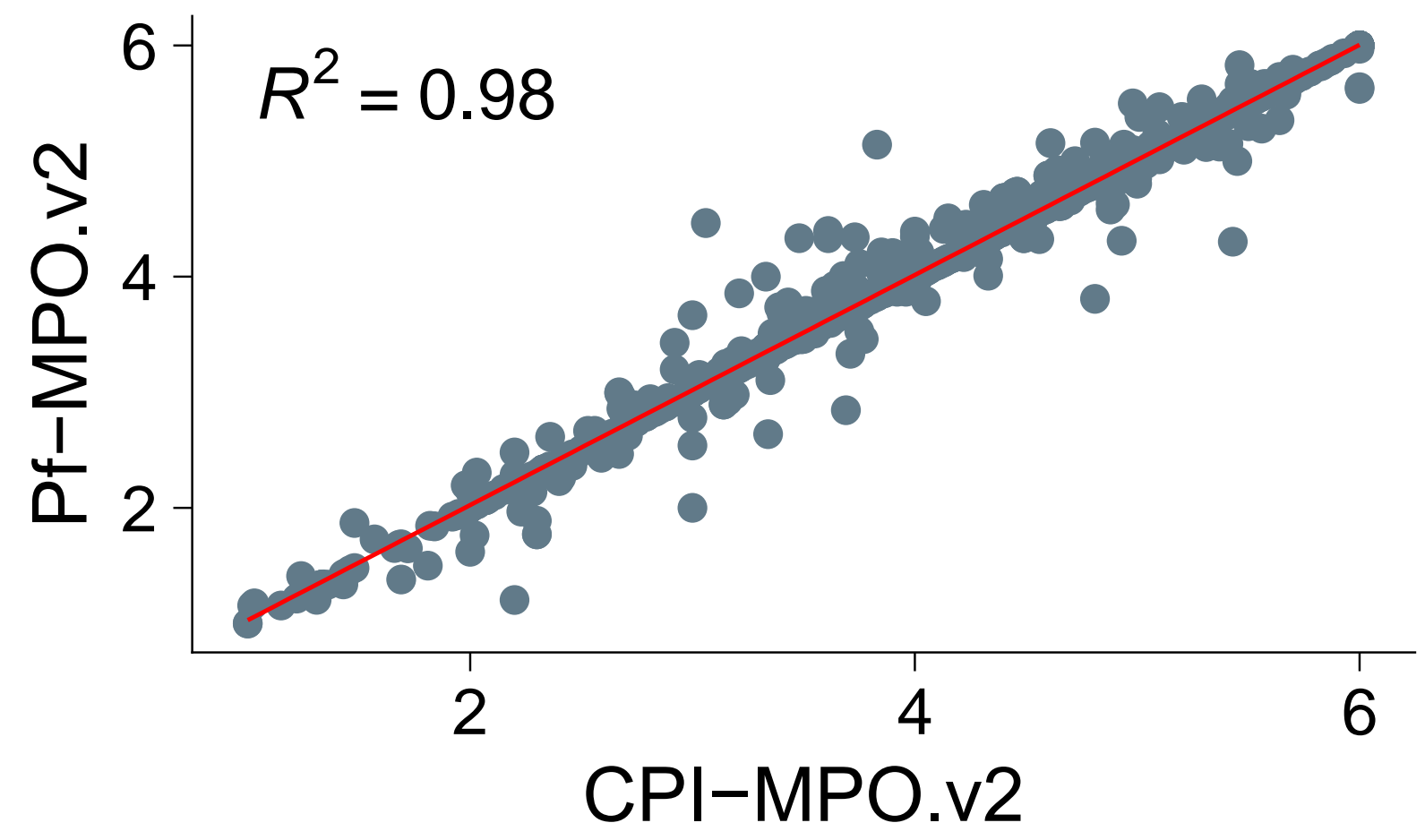

Figure S4. Comparison of MPO.v2 using descriptors generated from ChemAxon (CPI-MPO.v2) against their MPO.v2 as originally described ${ }^{2,3}$ (Pf-MPO.v2, $\mathbf{P}<<$ 0.005). 


\begin{tabular}{|c|c|c|c|c|c|c|}
\hline Pub_Name & Set & Target_Mechanism & MW & $\mathrm{Clog} \mathrm{P}$ & T0_ClogP & CNS-MPO \\
\hline Candidate-021 & Candidates & Enzyme & 315.4 & 3.1 & 0.97 & 5.1 \\
\hline Candidate-072 & Candidates & Dopaminergic GPCR & 360.4 & 3.1 & 0.97 & 5.0 \\
\hline Tramadol & Drugs & Reuptake Inhibitors & 263.4 & 3.1 & 0.95 & 4.6 \\
\hline Candidate-033 & Candidates & Opioid Receptor & 426.6 & 3.1 & 0.95 & 4.0 \\
\hline Candidate-092 & Candidates & Reuptake Inhibitors & 360.3 & 3.1 & 0.95 & 5.7 \\
\hline Candidate-052 & Candidates & Enzyme & 385.3 & 3.1 & 0.94 & 4.6 \\
\hline Citalopram & Drugs & Serotonergic GPCR & 324.4 & 3.1 & 0.93 & 5.0 \\
\hline Escitalopram & Drugs & Serotonergic GPCR & 324.4 & 3.1 & 0.93 & 5.0 \\
\hline Ropivacaine & Drugs & & 274.4 & 3.2 & 0.92 & 5.0 \\
\hline Minaprine & Drugs & Enzyme & 298.4 & 3.2 & 0.91 & 5.7 \\
\hline Quazepam & Drugs & Gaba Receptors & 386.8 & 3.2 & 0.90 & 3.7 \\
\hline Candidate-020 & Candidates & Glutamate receptors & 402.5 & 3.2 & 0.90 & 5.3 \\
\hline Amfebutamone & Drugs & Dopaminergic GPCR & 239.7 & 3.2 & 0.89 & 4.5 \\
\hline Riluzole & Drugs & Reuptake Inhibitors & 234.2 & 3.2 & 0.88 & 5.0 \\
\hline Candidate-085 & Candidates & Reuptake Inhibitors & 294.4 & 3.2 & 0.88 & 5.5 \\
\hline Candidate-104 & Candidates & Peptide GPCR & 419.6 & 3.2 & 0.88 & 4.0 \\
\hline Reboxetine & Drugs & Reuptake Inhibitors & 313.4 & 3.3 & 0.87 & 5.5 \\
\hline Venlafaxine & Drugs & Serotonergic GPCR & 277.4 & 3.3 & 0.87 & 4.7 \\
\hline Tacrine & Drugs & Enzyme & 198.3 & 3.3 & 0.86 & 4.5 \\
\hline Fluvoxamine & Drugs & Serotonergic GPCR & 318.3 & 3.3 & 0.84 & 4.6 \\
\hline Candidate-014 & Candidates & Opioid Receptor & 396.5 & 3.3 & 0.84 & 4.6 \\
\hline
\end{tabular}

Table S1: Part of the supplementary table adapted from Wager et al., ${ }^{1}$. 


\begin{tabular}{|c|c|c|c|c|}
\hline Models & AUC & Specificity & Selectivity & Accuracy (\%) \\
\hline Gao et al., (2017) ${ }^{4}$ & 0.85 & 0.77 & 0.99 & 89 \\
\hline Castillo-Garit $(2017)^{5}$ & ND & 0.88 & 0.85 & 86 \\
\hline Wang et al (2018) ${ }^{6}$ & 0.90 & 0.83 & 0.98 & 94 \\
\hline Plisson and Piggott (2019) ${ }^{7}$ & 0.78 & ND & ND & 82 \\
\hline Shaker et al., (2020) ${ }^{8}$ & 0.94 & 0.77 & 0.99 & 89 \\
\hline Singh et al., (2020) ${ }^{9}$ & ND & 0.71 & 0.92 & 87 \\
\hline This study* & 0.94 & 0.83 & 0.92 & 90 \\
\hline
\end{tabular}

Table S2. Comparison of recent BBB model statistics compared with the current Bayesian model which used 5 -fold cross validation and used the previously published dataset ${ }^{6}$. 


\section{Supplemental References}

1. Wager, T. T.; Hou, X.; Verhoest, P. R.; Villalobos, A., Moving beyond rules: the development of a central nervous system multiparameter optimization (CNS MPO) approach to enable alignment of druglike properties. ACS Chem Neurosci 2010, 1 (6), 435-49.

2. Rankovic, Z., CNS Physicochemical Property Space Shaped by a Diverse Set of Molecules with Experimentally Determined Exposure in the Mouse Brain. $J$ Med Chem 2017, 60 (14), 5943-5954.

3. Rankovic, Z., Retraction of "CNS Physicochemical Property Space Shaped by a Diverse Set of Molecules with Experimentally Determined Exposure in the Mouse Brain". J Med Chem 2019, 62 (3), 1699.

4. Gao, Z.; Chen, Y.; Cai, X.; Xu, R., Predict drug permeability to blood-brain-barrier from clinical phenotypes: drug side effects and drug indications. Bioinformatics 2017, 33 (6), 901-908.

5. Castillo-Garit, J. A.; Casanola-Martin, G. M.; Le-Thi-Thu, H.; Pham-The, H.; Barigye, S. J., A Simple Method to Predict Blood-Brain Barrier Permeability of Drug- Like Compounds Using Classification Trees. Med Chem 2017, 13 (7), 664-669.

6. Wang, Z.; Yang, H.; Wu, Z.; Wang, T.; Li, W.; Tang, Y.; Liu, G., In Silico Prediction of Blood-Brain Barrier Permeability of Compounds by Machine Learning and Resampling Methods. ChemMedChem 2018, 13 (20), 2189-2201. 
7. Plisson, F.; Piggott, A. M., Predicting Blood(-)Brain Barrier Permeability of MarineDerived Kinase Inhibitors Using Ensemble Classifiers Reveals Potential Hits for Neurodegenerative Disorders. Mar Drugs 2019, 17 (2).

8. Shaker, B.; Yu, M. S.; Song, J. S.; Ahn, S.; Ryu, J. Y.; Oh, K. S.; Na, D., LightBBB: Computational prediction model of blood-brain-barrier penetration based on LightGBM. Bioinformatics 2020.

9. Singh, M.; Divakaran, R.; Konda, L. S. K.; Kristam, R., A classification model for blood brain barrier penetration. J Mol Graph Model 2020, 96, 107516. 